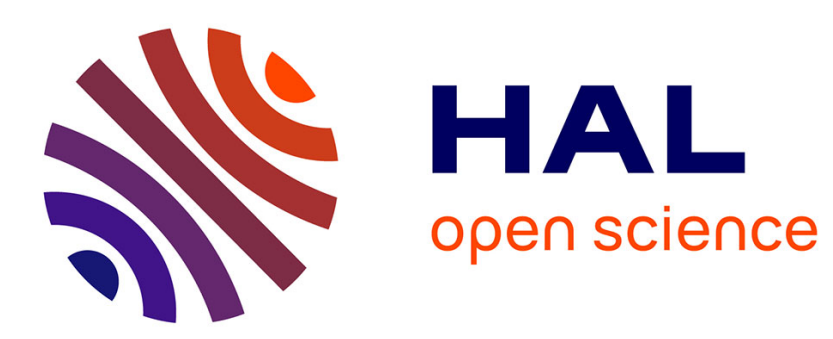

\title{
Rayonnement acoustique de coques élastiques étudié par des méthodes intégrales
}

\author{
A. Trad
}

\section{To cite this version:}

A. Trad. Rayonnement acoustique de coques élastiques étudié par des méthodes intégrales. Journal de Physique IV Proceedings, 1994, 04 (C5), pp.C5-877-C5-880. 10.1051/jp4:19945191 . jpa-00252874

\section{HAL Id: jpa-00252874 https://hal.science/jpa-00252874}

Submitted on 1 Jan 1994

HAL is a multi-disciplinary open access archive for the deposit and dissemination of scientific research documents, whether they are published or not. The documents may come from teaching and research institutions in France or abroad, or from public or private research centers.
L'archive ouverte pluridisciplinaire HAL, est destinée au dépôt et à la diffusion de documents scientifiques de niveau recherche, publiés ou non, émanant des établissements d'enseignement et de recherche français ou étrangers, des laboratoires publics ou privés. 


\title{
Rayonnement acoustique de coques élastiques étudié par des méthodes intégrales
}

\author{
A. TRAD
}

L.M.M.C., Tour 66 5ème étage, Université P. et M. Curie, Paris 6, 4 place Jussieu, 75252 Paris cedex 05, France

\begin{abstract}
A surface Helmholtz integral system is used for obtaining the solution of the exterior steady-state acoustic radiation problem for an elastic shell. It leads to the solution of a system of eight coupled integral equations governing two pressions, three components of the displacement vector on the exterior surface of the shell and three components of the displacement vector on its interior surface. The numerical method used to compute these pressions and components is tested on the case of a spherical shell in a radial field.
\end{abstract}

\section{INTRODUCTION}

Les problèmes de rayonnement acoustique de structures élastiques font 1 ' objet, depuis quelques années, de très nombreuses études théoriques, numériques et expérimentales. Leurs applications pratiques sont importantes pour la détection sous marine ou aérienne.

Dans ce travail nous nous intéressons au problème de la prévision du rayonnement acoustique d'une coque élastique finie, plongée dans un fluide extérieur dense, lorsqu'elle est insonée par un système d'ondes incidentes harmoniques.

Les équations générales - aux dérivées partielles - qui gouvernent les pressions, $p$ extérieure rayonnée, $\mathrm{p}_{\mathrm{i}}$ dans le fluide intérieur de la coque, et les déplacements sur les surfaces extérieure et intérieure de celle-ci, sont écrites explicitement. Pour résoudre ce système, une méthode intégrale simple - de type Helmholtz - est utilisée. Elle conduit à un système de huit équations intégrales couplées pour les huit inconnues da problème. Comme les structures élastiques considérées ici sont, en général, à symétrie de révolution, ce système intégral est discrétisé par une méthode de différences finies, avec une attention particulière portée au problème des singularités. Celles-ci sont traitées par une méthode de Ramberg à double précision. La méthode est testée sur le cas d'une coque sphérique plongée dans un champ incident radial variable, cas pour lequel la solution théorique exacte peut être écrite.

\section{POSTTION DU PROBLEME}

Considérons une coque tridimensionnelle finie $\Omega_{s}$, élastique et homogène, de coefficients de Lamé $\lambda$ et $\mu$ constants et de densité $\rho_{s}$. Supposée contenir un fluide interne de densité $\rho_{i}$ occupant le domaine intérieur $\Omega_{\mathrm{i}}$, elle est plongée dans un fluide externe de densité $\rho_{\mathrm{e}}$, occupant le domaine extérieur illimité $\Omega_{\mathrm{e}}$. Les deux fluides sont supposés parfaits et 
incompressibles. Les deux surfaces $\Sigma_{\mathrm{e}}$ extérieure et $\Sigma_{\mathrm{i}}$ intérieure de la coque sont supposées régulières, admettant une dérivée normale extérieure notée $n$.

Cette coque est insonée par un train d'ondes harmoniques planes, de fréquence réelle $\omega$. Désignons par $p_{\text {inc }}$ le champ de pression incident, auquel correspond le champ de déplacement $u_{i n c}$; et supposons la dépendance en temps en $\exp (-\mathrm{i} \omega t)$. Deux types d'ondes sont induits dans le fluide extérieur:

.. un champ de diffraction dû à la diffraction du champ incident sur la structure supposée rigide. Sa partie stationnaire est gouvernée par un problème de Newman extérieur.

.. un champ de radiation dû aux vibrations de la structure élastique

La somme de ces deux champs, appelée champ rayonné, fait l'objet de cette étude.

\section{EQUATIONS GENERALES DU PROBLEME}

Pour une structure élastique à surface $S$ régulière, insonée par un champ de fréquence réelle, il est bien connu [1] que le système qui gouverne la pression rayonnée à l'extérieur et les déplacements dáns cette structure admet une solution unique régulière; si les conditions aux limites sont continues sur $\mathrm{S}$.

Pour la coque élastique considérée ici, notons $p, p$ et $u$ la pression rayonnée dans le fluide extérieur, la pression dans le fluide intérieur et le déplacement dans la coque, respectivement Leurs parties stationnaires - avec les mềmes notations - sont gouvernées par le système suivant noté $\left(S_{A}\right)$

. L'équation de Helmholtz et la condition de radiation de Sommerfeld pour p:

$$
\begin{aligned}
& \left(\Delta+\mathrm{k}^{2} \mathrm{p}\right)=\mathrm{O} \quad \text { dans } \Omega_{e} \\
& |(\partial \mathrm{p} / \partial \mathrm{r})-\mathrm{ikp}|=\mathrm{O}\left(\mathrm{r}^{-2}\right) ;|\mathrm{p}|==\mathrm{O}\left(\mathrm{r}^{-1}\right), \quad \text { pour } \mathrm{r}=|\mathrm{x}| \rightarrow \infty
\end{aligned}
$$

L'équation de Navier pour les déplacements dans la structure:

$$
\rho_{s} \omega^{2} u+\Delta^{*} u=0 \quad \text { dans } \Omega_{s}
$$

$\Delta^{*}=(\lambda+\mu) \operatorname{grad}$ div $+\mu \Delta$ est l'opérateur dé Navier.

- avec les conditions aux limites suivantes, exprimant, - à l'interface structure-fluide, la conservation de la composante normale du déplacement et la continuité du tenseur des contraintes:

$$
\begin{aligned}
& \partial p / \partial n=\rho_{\mathrm{s}} \omega^{2} \text { u.n }-f_{1} \quad \text { sur } \Sigma_{e} \quad f_{1}=\rho_{e} \omega^{2} u_{i n c} \cdot n \\
& \text { (5) } \quad \sigma(u) n=-p n-f_{2} \text { sur } \Sigma_{e} \quad f_{2}=-p_{\text {inc }} n
\end{aligned}
$$

une équation de Helmholtz pour la pression dans $\Sigma_{\mathbf{i}}$ :

$$
\left(\Delta+k_{i}^{2} p_{i}\right)=0 \quad \text { dans } \Omega_{i}
$$

avec les conditions aux limites:

$$
\begin{aligned}
& \partial p_{i} / \partial n=\rho_{s} \omega^{2} u_{i} \cdot n=\rho_{s} \omega^{2} u . n \quad \text { sur } \Sigma_{i} \\
& \sigma(u) n=-p_{i} n \quad \text { sur } \Sigma_{i}
\end{aligned}
$$


et enfin, la condition:

(9) $\quad \mathrm{p}_{\mathbf{i}} \quad$ finie partout dans le domaine fluide intérieur $\Sigma_{\mathrm{j}}$

Dans le système $S_{1}, \sigma$ est le tenseur classique des contraintes; $k$ et $k_{j}$ sont les nombres d'onde, $k=(\omega / c)$ et $k_{i}=\left(\omega / c_{i}\right)$, c et $c_{i}$ étant les vitesses du son dans les fluides exiérieur et intérieur, respectivement.

\section{METHODE DE RESOLUTION - REPRESENTATION INTEGRALE}

Pour une coque de forme géométrique quelconque, il est évidernment impossible d'obtenir une solution analytique du système $\left(S_{1}\right)$. Numériquement sa résolution n'est pas aisée problème du second ordre et condition de Sommerfeld, notamment - d'où le recours à des représentations des solutions de ce système. Pour ce faire, les représentations intégrales sont très utilisées. Dans ce travail nous avons utilisé:

.. la méthode de l'intégrale de surface de Helmholtz pour les équations des ondes réduites qui gouvernent les pressions dans les fluides,

.. l'égalité de Betti, [2], pour les équations du mouvement qui gouvernent les composantes du déplacement.

En tenant compte des conditions aux limites on est ainsi amené, pour les pressions et les déplacements, à un système $\left(S_{2}\right)$ de 8 équations intégrales couplées pour les 8 inconnues du problème, la pression rayonnée $p$, la pression intérieure $p_{i}$, les 3 composantes $u_{j} j=1,2,3 \mathrm{du}$ déplcement sur la surface extérieure et sur la surface intérieure de la coque. Posons:

$$
g(x, y)=\exp (i k|x-y|) / 4 \pi|x-y| ; \quad g_{i}(x, y)=\exp \left(i k_{i}|x-y|\right) / 4 \pi|x-y|
$$

le système $\left(S_{2}\right)$ à résoudre s'écrit:

$$
\begin{aligned}
& (1 / 2) p(x)=F_{0}+\int_{\Sigma_{e}}\left[p(\partial g(x, y) / \partial n(y))-\rho_{e} \omega^{2} g u . n\right] d \Sigma_{e} \quad x \in \Sigma_{e} \\
& (1 / 2) u_{j}(x)=-F_{j}-\int_{S}\left[p G^{j} n+T G^{j} . n\right] d \Sigma_{e} \quad x \in S, \quad S=\Sigma_{e} \cup \Sigma_{i} \\
& \int_{\Sigma i}\left[p_{i}\left(\partial g_{i}(x, y) / \partial n(y)\right)-\rho_{i} \omega^{2} g_{i} u . n\right] d \Sigma_{i}=0 \quad x \in \Sigma_{i}
\end{aligned}
$$

Dans ce système intégral de type Fredhom de seconde espèce, les termes libres $F_{k} k=0,1,2,3$ fonctions des données, sont connus; et [2] le vecteur $\mathrm{G}^{\mathrm{J}}$ est donné par:

$$
\begin{aligned}
& \mathrm{G}^{\mathrm{J}}=\left(1 / \rho_{\mathrm{s}} \omega^{2}\right)\left[\operatorname{rot} \operatorname{rot} \mathrm{W}^{\mathrm{J}}-\operatorname{grad} \operatorname{div} \mathrm{V}^{\mathrm{J}}\right] ; \quad \mathrm{j}=1,2,3 \\
& \mathrm{~W}^{\mathrm{J}}(\mathrm{x}, \mathrm{y})=(1 / 4 \pi|\mathrm{x}-\mathrm{y}|) \delta^{\mathrm{J}} \exp \left(\mathrm{ik}_{\mathrm{T}}|\mathrm{x}-\mathrm{y}|\right) ; \quad \mathrm{k}_{\mathrm{T}}=\sqrt{\rho_{\mathrm{s}} \omega^{2} / \mu} \\
& \mathrm{V}^{\mathrm{J}}(\mathrm{x}, \mathrm{y})=(1 / 4 \pi|\mathrm{x}-\mathrm{y}|) \delta^{\mathrm{J}} \exp \left(\mathrm{ik}_{\mathrm{L}}|\mathrm{x}-\mathrm{y}|\right) ; \quad \mathrm{k}_{\mathrm{L}}=\sqrt{\rho_{\mathrm{s}} \omega^{2} /(\lambda+2 \mu)}
\end{aligned}
$$

le vecteur $\delta^{\mathrm{J}}$ ayant pour composantes : $\left(\delta^{\mathrm{J}}\right)_{\mathrm{i}}=\delta_{\mathrm{ij}}$ symbole de Kronecker, $\mathrm{i}=1,2,3$

La contrainte tangentielle $\mathrm{T}$ est définie par: $\mathrm{Tu}=\sigma(\mathrm{u}) \mathrm{n}$

Notons que, une fois le système $\left(\mathrm{S}_{2}\right)$ résolu, c'est à dire que lorsque la pression et les composantes du déplacement sont déterminées sur la surface extérieure de la coque, la relation (10) - sans le facteur (1/2) - donne simplement ( pas de discontinuités) et directement la pression rayonnée en tout point du fluide extérieur. 
Remarque: Il est bien connu [3] [4], que, pour une structure rigide, la méthode intégrale utilisée ici, comme celles de simple ou de double couche, n'est pas valable pour tous les nombres d'onde réels du champ incident. Elle échoue pour des nombres d'onde dits irréguliers, valeurs propres d'un problème de Dirichlet intérieur. Il en est de même pour une structure élastique [5]. $D^{\prime}$ autres méthodes peuvent être employées pour éviter ces difficultés [6].

\section{RESOLUTION NUMERIQUE}

En vue des applications à la forme LINE, corps cylindrique terminé à ses deux bouts par deux hémisphères, nous avons considéré une coque de révolution. La discrétisation du système $\left(S_{2}\right)$ par une méthode de différences finies simple, conduit à un système algébrique linéaire du type $\mathrm{A} X=\mathrm{B}$. Le vecteur $\mathrm{B}$, dépendant du champ incident, est connu; la matrice A est à coefficients complexes, ses termes diagonaux sont singuliers; ils sont traités par une méthode de Ramberg à double précision performante. Le système algébrique est traité par une méthode de Jordan à pivot total.

\section{TEST DE LA METHODE - LE CAS D'UNE COQUE SPHERIQUE}

Le schéma numérique utilisé est testé sur le cas d'une coque sphérique homogène en acier, plongée dans un champ acoustique radial non uniforme; cas pour lequel on peut écrire la solution théorique exacte. les calculs numériques sont effectués pour des nombres d'onde $\mathbf{k}$ réguliers, faibles ou moyens - $\mathrm{ka}$, a rayon de la sphère extérieure, variant de 0.5 à 5 . Les calculs effectués sur une station HP Apollo 400 sont rapides et donnent des résultats satisfaisants qui seront exposés et commentés au congrès.

\section{REFERENCES}

[1] Sanchez-Hubert, J. et Sanchez-Palencia, E. Vibration and coupling of continuous systems. Asymptotic methods. Springer, Berlin (1989).

[2] Kupradze, V.D. Potential methods in the theory of elasticity. I.P.F.S.T., Jérusalem,(1965).

[3] Copley, L.G. Fundamental results concerning integral representations in acoustic radiation. J. Acoust. Soc. Amer. 44 (1968), 28-32.

[4] Schenck, H.A. Improved integral formulation for acoustic radiation problems. J. Acoust. Soc. Amer. 44 (1968), 41-68.

[5] Trad, A. A surface Helmholtz integral system for a sound radiation by an elastic body. A nonuniqueness theorem. Int. J. Engng. Sci. 29,10 (1991), 1333-1335.

[6] Trad, A. Rayonnement de surfaces rigides dans un champ acoustique harmonique à toutes les fréquences réelles. Acustica 76 (1992), 73-83. 